Thies Schulze, Paul J. Weldon and Stefan Schulz*

\title{
Scent gland constituents of the Middle American burrowing python, Loxocemus bicolor (Serpentes: Loxocemidae)
}

DOI 10.1515/znc-2017-0006

Received January 13, 2017; revised February 14, 2017; accepted February 14, 2017

\begin{abstract}
Analysis by gas chromatography/mass spectrometry of the scent gland secretions of male and female Middle American burrowing pythons (Loxocemus bicolor) revealed the presence of over 300 components including cholesterol, fatty acids, glyceryl monoalkyl ethers, and alcohols. The fatty acids, over 100 of which were identified, constitute most of the compounds in the secretions and show the greatest structural diversity. They include saturated and unsaturated, unbranched and mono-, di-, and trimethyl-branched compounds ranging in carbonchain length from 13 to 24 . The glyceryl monoethers possess saturated or unsaturated, straight or methylbranched alkyl chains ranging in carbon-chain length from 13 to 24. Alcohols, which have not previously been reported from the scent glands, possess straight, chiefly saturated carbon chains ranging in length from 13 to 24 . Sex or individual differences in secretion composition were not observed. Compounds in the scent gland secretions of L. bicolor may deter offending arthropods, such as ants.
\end{abstract}

Keywords: alcohols; fatty acids; glyceryl monoalkyl ethers; scent gland; snake.

Dedication: In memory of the late Lothar Jänicke

\footnotetext{
*Corresponding author: Stefan Schulz, Institut für Organische Chemie, Technische Universität Braunschweig, Hagenring 30, 38106 Braunschweig, Germany, E-mail: stefan.schulz@tu-bs.de. http://orcid.org/0000-0002-4810-324X

Thies Schulze: Institut für Organische Chemie, Technische Universität Braunschweig, Hagenring 30, 38106 Braunschweig, Germany

Paul J. Weldon: Smithsonian Conservation Biology Institute, National Zoological Park, 1500 Remount Road, Front Royal, Virginia 22630, USA
}

\section{Introduction}

Reptiles possess a number of macroscopic integumentary glands, many of which are unique to a particular order or suborder (see [1] for recent bibliography). Snakes (order Squamata, suborder Serpentes) possess a paired exocrine organ, called the scent gland, situated in the base of the tail and opening through two ducts at the margin of the cloacal orifice. Foul-smelling fluids typically are released from this gland when snakes are molested, thus inspiring the frequent suggestion that these secretions repel predators. Other proposed functions for the scent glands include the production by females of courtship deterrents against unpreferred suitors $[2,3]$.

Chemical analyses reveal that scent gland secretions consist chiefly of proteins [4-6]. Lipids and other lowmolecular components, including volatiles that impart the characteristic secretion odors of some species, are also present. Cholesterol [6-11], which is a common tetrapod skin lipid, and carboxylic acids [5-10, 12] are widely documented in scent gland secretions. Nitrogen-containing compounds, including piperidone [12], amines [8, 12], and amides $[8,9]$, also have been indicated in some taxa. 1-O-Monoalkylglycerols have been reported in the secretions of the western diamondback rattlesnake (Crotalus atrox) [11].

The Middle American burrowing python (Loxocemus bicolor Cope) occurs in moist to dry forests from southwestern Mexico through Guatemala, Honduras, El Salvador, Nicaragua, and into Costa Rica (Figure 1). This fossorial snake feeds predominantly on small vertebrates and reptile eggs $[13,14]$. Loxocemus bicolor is the sole member of its genus and family, the Loxocemidae. Molecular studies indicate that this snake is a basal alethinophidean, closely allied with or properly placed within the Pythonidae $[15,16]$. We chose to investigate the scent gland secretions of L. bicolor because of its unique phylogenetic position in a clade of primitive constricting snakes. This secretion proved to be particularly diverse, consisting of more than 300 lipid components. 


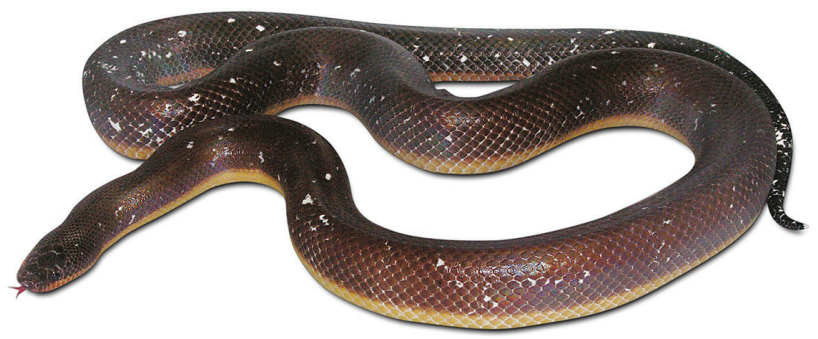

Figure 1: An adult female Loxocemus bicolor (total length $=90 \mathrm{~cm}$ ). Photograph by T. Barker (Vida Preciosa International, Inc., Boerne, TX, USA).

\section{Results}

The analysis of the scent gland secretions revealed the presence of many peaks in the total ion chromatograms. The bad peak shapes leading to largely overlapping peaks suggested the presence of a high percentage of compounds with active hydrogens, such as alcohols and acids (Figure 2). Therefore, the samples were analyzed again after derivatization with $N$-methyl- $N$-(trimethylsilyl) trifluoroacetamide (MSTFA, Figure 3). This procedure led to better peak separation and allowed the identification of the major compound classes. Over 300 components were observed. Cholesterol was a major constituent of all samples. The other components belonged to the following compound classes, in order of abundance: fatty acids, glyceryl monoalkyl ethers, and alcohols.

\subsection{Fatty acids}

The acids comprise most of the compounds in the secretions and showed the highest structural diversity. For GC/ MS analysis of these acids, the crude extracts were treated with diazomethane to yield the corresponding methyl esters, which improved gas chromatographic behavior

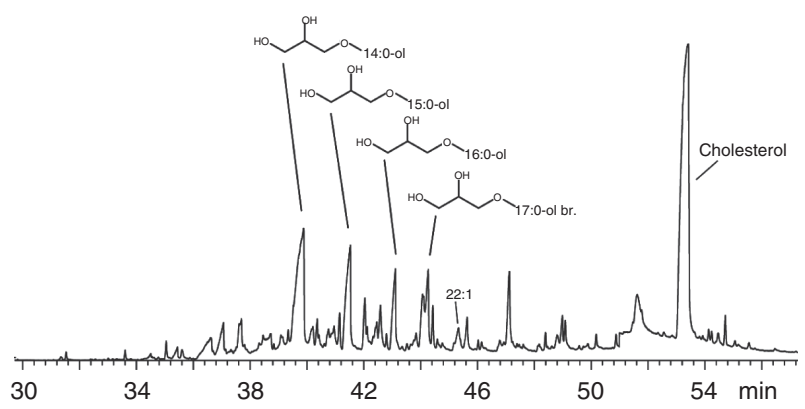

Figure 2: Total ion chromatogram of an extract of the scent gland secretions of Loxocemus bicolor.

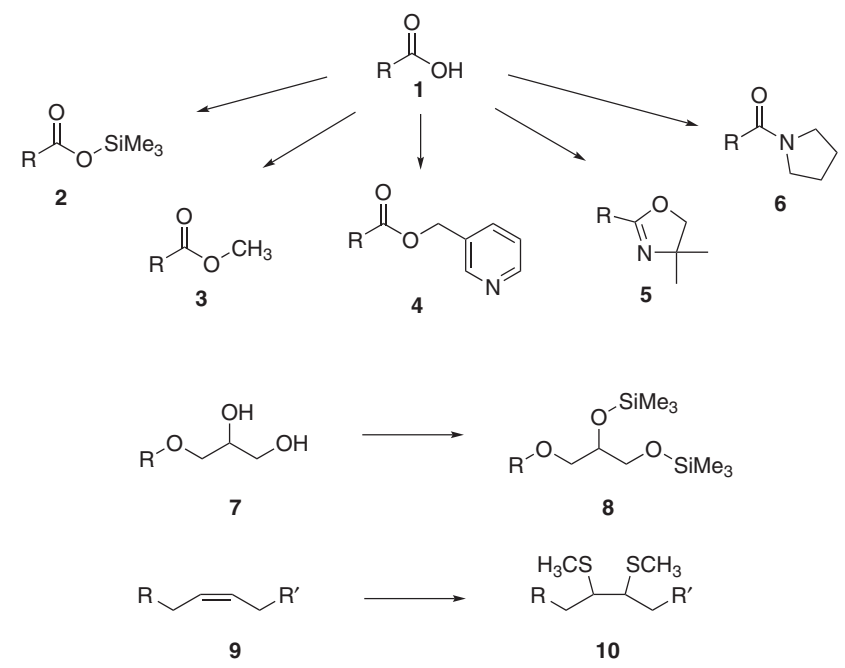

Figure 3: Derivatization reactions performed with extracts for structure elucidation.

(Figure 3). Mass spectra of methyl esters (3) allow the detection of several structural features such as methyl groups near the ester group [17] and the number of double bonds in the chain. The retention index $I$ can be used to identify methyl groups near the alkyl end of the chain [18]. Nevertheless, internal methyl groups or double-bond positions cannot be determined, and other derivatives must be used to locate such groups. The best results are usually obtained by converting of the acids into 3-pyridiylmethyl esters 4 [19], but other methods such as the formation of dimethyloxazolines 5 [20] or pyrrolidides 6 [21] are also used.

A major problem in the analysis of the snake acids proved to be the large number of compounds present. This led to extensive coelution of compounds, making proper identification difficult. All three derivatization procedures mentioned were performed. It turned out that the analysis of both 3-pyridiylmethyl esters and pyrrolidides were necessary for proper identification, while dimethyloxazolines did not add additional information. To complement this approach, dimethyl disulfide derivatization was used to assign the position of double bonds in addition to 3-pyridiylmethyl esters.

As an example, the identification of a major methylbranched acid, 14-methyloctadecanoic acid, and a major unsaturated acid, 11-icosenoic acid will be discussed in detail. The various spectra of their derivatives are shown in Figure 4 as examples. These spectra allow the localization of the methyl branch and the location of the double bond in the two compounds. While the spectrum of methyl 14-methyloctadecanoate (Figure 4A) is difficult to interpret a priori [17], the respective pyrrolidide (Figure 
A

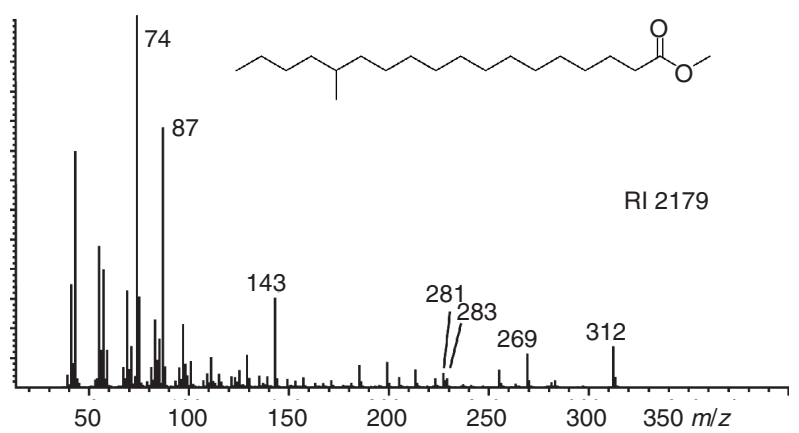

C

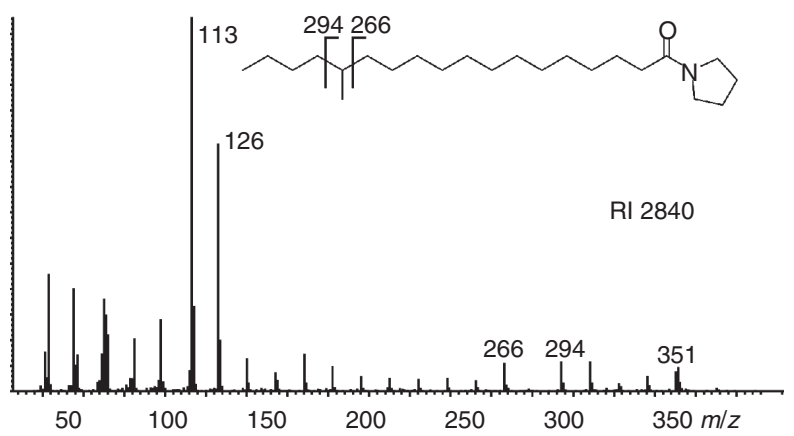

E

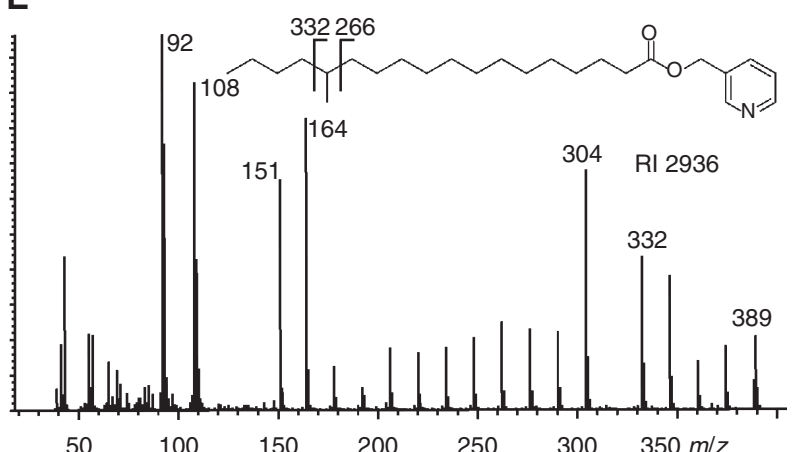

B

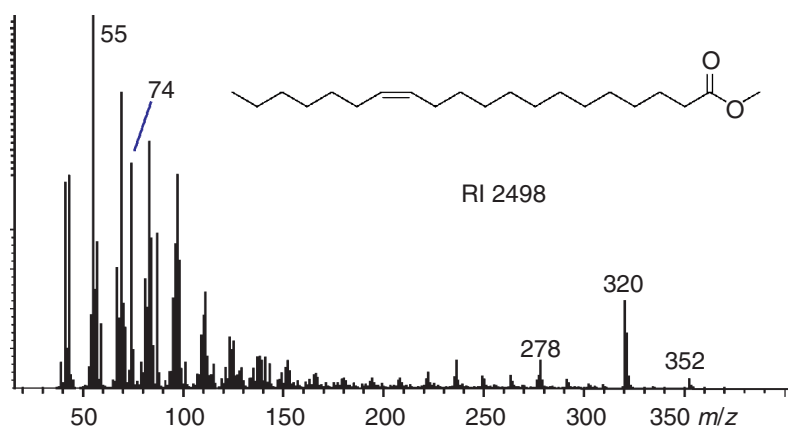

D

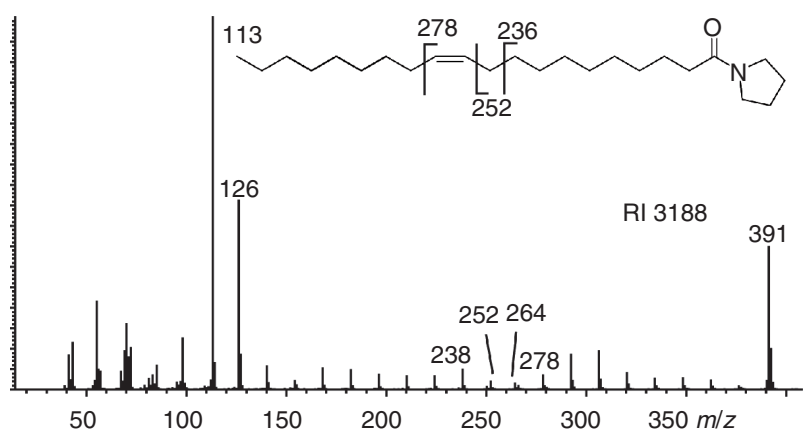

$\mathbf{F}$

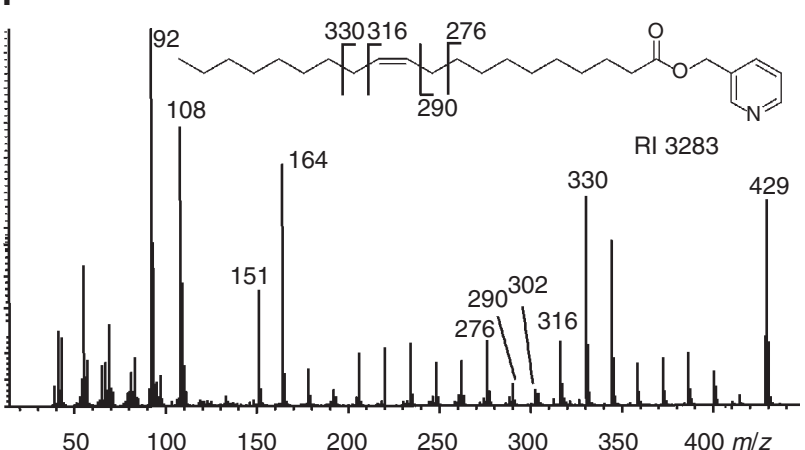

Figure 4: Mass spectra of derivatives of 14-methyloctadecanoic (A, C, E) and 11-icosenoic acids (B, D, F), gas chromatographic retention indices and characteristic mass spectrometric cleavages. Methyl esters (A, B), pyrrolidides(C, D), and 3-pyridylmethyl esters (E, F).

4C) lacks ion $m / z 280$ of the ion series $\left[\mathrm{C}_{4} \mathrm{H}_{8} \mathrm{NCOC}_{n} \mathrm{H}_{2 n}\right]^{+}$. This ion series is formed by sequential alkyl cleavage along the chain. Similarly, the respective 3-pyridiylmethyl ester (Figure 4E) lacks ion $\mathrm{m} / z 318$ of the ion series $\left[\mathrm{C}_{6} \mathrm{H}_{7} \mathrm{NOCOC}_{n} \mathrm{H}_{2 n}\right]^{+}$. The intensities of the neighboring ions in these homologous series $(\mathrm{m} / z$ 266/294 and $m / z$ 304/332, respectively) are enhanced. The gaps in the ion series indicate a methyl branch in the chain that can be located by the ions mentioned at C-14.

The localization of the double bond is more difficult but can also be achieved. The methyl ester (Figure 4B) mass spectrum does not give any hints on the localization of the double bond. The mass spectrum of the pyrrolidide shows 14 amu gaps between the methylene groups, but only 12 amu between $\mathrm{m} / \mathrm{z} 252$ and 264 (Figure 4D). Obviously, the double bond is near C-11 but cannot exactly be located. Somewhat better results are obtained with the 3-pyridiylmethyl esters (Figure 4E). The strong allylic cleavage ion at $\mathrm{m} / \mathrm{z} 330$ and the 12 amu gap between $\mathrm{m} / z 290$ and 302 locates the double bond at C-11. Nevertheless, a good and clean spectrum is required for reliable identification. This was not always the case for the other acids of the secretion. The most reliable results were obtained by the mass spectra of dimethyl disulfide (DMDS) adducts (10) of the unsaturated methyl esters [22, 23]. 11-Icosenoic acid was identified because the mass spectrum of the DMDS adduct of its methyl ester furnished prominent ions at $\mathrm{m} / z 245\left[\mathrm{CH}_{3} \mathrm{SC}_{10} \mathrm{H}_{20} \mathrm{CO}_{2} \mathrm{CH}_{3}{ }^{+}\right]$, 
$213\left[\mathrm{CH}_{3} \mathrm{SC}_{10} \mathrm{H}_{20} \mathrm{CO}-\mathrm{CH}_{3} \mathrm{OH}^{+}\right]$and $173\left[\mathrm{C}_{9} \mathrm{H}_{20} \mathrm{SCH}_{3}^{+}\right]$[21]. Two isomers, 12-icosenoic acid, characterized by $\mathrm{m} / \mathrm{z}$ 259 , 227, and 159, as well as 13 -icosenoic acid, characterized by $m / z 273,241$, and 145 , were also present in the secretion.

The individual acids were identified by interpretation of the mass spectra of the derivatives as just discussed, according to well established rules [22, 24-29], and by correlation with gas chromatographic retention indices $I$ $[18,30]$. Over 100 acids were identified. The acids of L. bicolor chiefly consist of long-chain, unbranched, saturated, and unsaturated acids, as well as mono-, di-, and trimethyl-branched acids (Table 1). Major constituents are 13-docosenoic acid, docosanoic acid, 15-tricosenoic acid, 15-tetracosenoic acid, and 14-methylhexadecanoic acid. Methyl branching occurs predominately at C-4, C-10, $\mathrm{C}-12$, and C-14. Double bonds prevail at the $\omega-9$ position, a typical location for fatty acids of animal origin. A second double bond can occur in very long tetracosadienoic acids. The two acids F99 and F101 (Table 1) represent the two acid types present, those with remote double bonds and those with bishomoconjugated double bonds. Structures of minor amounts of similar acids could not be fully assigned.

Acids with both methyl branches and double bonds occur as well but in minor amounts. Even-numbered carbon chains are dominating, although odd-numbered acids also occur. 2,3-Dihydrofarnesoic acid is the only acid likely originating from the terpene biosynthetic pathway. No attempt was made to elucidate the configuration of the double bonds, but the common (Z)-configuration seems most likely. Structures of some of the acids are shown in Figure 5.

A general biosynthetic model explaining the diversity of the acids is shown in Figure 6. Fatty acids are biosynthesized starting from acetyl-coenzyme A (12) that is elongated by malonate (15) until a typical chain length is reached, e.g. stearic acid (13). Dehydrogenation by a $\Delta$ 9-desaturase leads to oleic acid, (Z)-9-octadecenoic acid (16), a $\omega-9$ unsaturated acid (Figure 6). Several variations of this pathway obviously occur during biosynthesis of the scent gland acids. Replacement of one malonyl-unit with methylmalonate (14) during chain formation leads to methyl groups in the chain. An additional $\Delta 11$-desaturase furnishes $\omega-7$ unsaturated acids. The chain can be elongated by additional malonate units or biosynthesis can stop earlier, leading to shorter acids than $\mathrm{C}_{18^{\circ}}$. After elongation, a second desaturation by a $\Delta 7$-desaturase leads to dienoic acids. Finally, odd-numbered acids can be formed by using propionate-CoA (11) instead of $\mathbf{1 2}$ as chain starter, or alternatively, acids can be shortened by $\alpha$-oxidation by one carbon. Combining all these elements of chain-length diversity from $\mathrm{C}_{12}$ to $\mathrm{C}_{24}$ including oddnumbered acids, desaturation on a few positions, and methyl groups near the acid group and in the middle/end of the chain leads to the extraordinary diversity in acid structures.

Although variation in the relative proportions of individual compounds occur, neither the acids nor other compounds appeared to be sex specific; the secretion looks remarkably similar for males and females (Figure 7). Furthermore, the composition of the gland does not vary greatly; many compounds were present in all samples analyzed, despite their different origin. It seems, therefore, that the composition of the secretion is relatively stable.

\subsection{1-O-Alkylglycerols}

Glyceryl alkyl ethers in the scent gland secretions were identified by their mass spectra and the trimethylsilyl (TMS) derivatives 8. The mass spectrum of $1-O$-tetradecylglyceride and the respective TMS-ether is shown in Figure 8. The mass spectrum of the latter features a base peak at $\mathrm{m} / z 205\left[\left(\mathrm{CH}_{3}\right)_{3} \mathrm{SiOCH} \mathrm{CHOSi}_{2}\left(\mathrm{CH}_{3}\right)_{3}{ }^{+}\right]$, the ion $\mathrm{m} / z$ 147 indicating the presence of two TMS groups [31], and a small M-15 ion [11, 32]. The ion $\mathrm{m} / z 205$ clearly indicates that the terminal alcohol is used in an ether linkage, as do the ions $\mathrm{m} / z 61$ and 227 in the original spectrum. Over 40 ethers were present in the secretion in which a homologous series of unbranched, saturated ethers from $\mathrm{C}_{13}$ to $\mathrm{C}_{16}$ as well as 1-0-14-methylhexadecylglycerol dominated. They were accompanied by minor amounts of methylbranched or unsaturated ethers with longer chains (Table 2 and Figures 8 and 9). The locations of branching points or double-bond positions were not determined; however, iso- and anteiso-branched compounds were identified based on established rules for calculating their gas chromatographic retention indices $(I)$ in long-chain compounds $[18,30]$.

\subsection{Alcohols}

A homologous series of 1-alkanols were identified after derivatization with MSTFA. The chain length ranged from $\mathrm{C}_{13}$ to $\mathrm{C}_{24}$. Even-numbered alcohols were present in higher amounts than the odd-numbered alcohols, with increasing concentration according to chain length. No branched alcohols could be found; tetracosen-1-ol was identified as the only unsaturated alcohol. 
Table 1: Fatty acids occurring in the scent gland secretions of male (M) and female (F) Loxocemus bicolor. Relative proportion to the major acid $\mathbf{F 8 7}(100 \%)$.

\begin{tabular}{|c|c|c|c|c|c|c|c|c|c|c|c|c|}
\hline & & $\mathbf{R I}$ & $\begin{array}{l}\text { M1 } \\
\widehat{\sigma}\end{array}$ & $\begin{array}{l}\text { M2 } \\
\sigma^{2}\end{array}$ & $\begin{array}{c}\text { M3 } \\
\delta\end{array}$ & $\begin{array}{l}\text { F1 } \\
\text { 우 }\end{array}$ & $\begin{array}{l}\text { F2 } \\
\text { 우 }\end{array}$ & $\begin{array}{l}\text { F3 } \\
\text { 우 }\end{array}$ & $\begin{array}{r}\mathrm{F} 4 \\
\text { 우 }\end{array}$ & $\begin{array}{r}\text { F5 } \\
\text { 오 }\end{array}$ & $\begin{array}{r}\mathrm{F} 6 \\
\text { 우 }\end{array}$ & $\begin{array}{l}\text { F7 } \\
\text { 우 }\end{array}$ \\
\hline F1 & 3-Methyldodecenoic acid & 1572 & 2.1 & 2.1 & 1.2 & 2.0 & $x$ & 1.3 & 1.1 & 1.1 & 0.3 & 5.2 \\
\hline F2 & 3-Methyldodecenoic acid & 1681 & 0.6 & & & & 1.4 & 1.2 & 0.6 & 0.3 & & 2.0 \\
\hline F3 & 2,3-Dihydrofarnesoic acid & 1725 & 1.1 & 0.5 & 0.3 & 0.2 & 0.3 & 0.2 & 0.4 & 0.2 & 0.3 & 2.0 \\
\hline F4 & 2-Methyltetradecanoic acid & 1762 & 0.1 & & & & & & & & & $\operatorname{tr}$ \\
\hline F5 & 3-Methyltetradecanoic acid & 1772 & & & & & & & & & & $\operatorname{tr}$ \\
\hline F6 & 4-Methyltetradecanoic acid & 1778 & 0.7 & & & & & & & & 0.3 & 0.7 \\
\hline F7 & Pentadecanoic acid & 1830 & 0.6 & & & & 0.7 & & & & & \\
\hline F8 & 4,12-Dimethyltetradecanoic acid & 1850 & & & & & & & & & & 1.4 \\
\hline F9 & 2-Methylpentadecanoic acid & 1863 & & & & & & & & & & 1.0 \\
\hline F10 & 3-Methylpentadecanoic acid & 1868 & & & & & & & & & & 0.5 \\
\hline F11 & 6-Methylpentadecanoic acid & 1873 & & & & & & & & & & $\operatorname{tr}$ \\
\hline F12 & 8-Methylpentadecanoic acid & 1878 & & & & & & & & & & 1.0 \\
\hline F13 & 4-Methylpentadecanoic acid & 1880 & 4.6 & 4.0 & 5.9 & 3.2 & 6.6 & 4.6 & 4.4 & 1.4 & 4.5 & 9.6 \\
\hline F14 & 4,8,12-Trimethyltetradecanoic acid & 1883 & & & & & & & & 0.9 & 0.5 & 1.0 \\
\hline F15 & 12-Methylpentadecanoic acid & 1888 & & & & & & & & 0.7 & 0.6 & 1.9 \\
\hline F16 & 14-Methylpentadecanoic acid & 1894 & & & & & & & & 0.7 & & 1.6 \\
\hline F17 & 13-Methylpentadecanoic acid & 1902 & 0.6 & & & & & & & & & 2.4 \\
\hline F18 & Hexadecenoic acid & 1905 & 0.8 & & 1.8 & $\operatorname{tr}$ & 2.1 & $\operatorname{tr}$ & $\operatorname{tr}$ & & & 1.1 \\
\hline F19 & $2, x$-Dimethylpentadecanoic acid & 1914 & 0.7 & $\operatorname{tr}$ & 0.9 & $\operatorname{tr}$ & 1.2 & 1.1 & 1.2 & & 0.5 & 3.7 \\
\hline F20 & $2, x$-Dimethylpentadecanoic acid & 1920 & $\operatorname{tr}$ & & & & & & & & & 1.4 \\
\hline F21 & 4,x-Dimethylpentadecanoic acid & 1924 & 0.8 & $\operatorname{tr}$ & 0.9 & 0.9 & 0.9 & 1.3 & 1.2 & 0.4 & 0.5 & 4.0 \\
\hline F22 & Hexadecanoic acid & 1930 & 7.5 & 4.9 & 4.0 & 5.0 & 4.2 & 3.0 & 3.2 & 4.6 & 2.1 & 13.2 \\
\hline F23 & 4,12-Dimethylpentadecanoic acid & 1932 & $\operatorname{tr}$ & $\operatorname{tr}$ & $\operatorname{tr}$ & 0.8 & $\operatorname{tr}$ & 1.0 & 1.3 & 0.3 & 1.0 & 5.5 \\
\hline F24 & 2-Methylhexadecanoic acid & 1963 & 3.9 & 4.0 & 4.6 & 2.6 & 4.0 & 2.9 & 3.3 & 5.0 & 4.0 & 8.8 \\
\hline F25 & $4, x, x$-Trimethylpentadecanoic acid & 1969 & 0.9 & $?$ & 0.9 & 0.8 & $\operatorname{tr}$ & 1.1 & 1.0 & 0.5 & 0.5 & 2.5 \\
\hline F26 & 10-Methylhexadecanoic acid & 1974 & 13.9 & $\operatorname{tr}$ & 14.8 & 7.0 & 17.0 & 10.5 & 10.5 & 11.2 & 11.9 & 24.8 \\
\hline F27 & 4-Methylhexadecanoic acid & 1979 & 13.2 & 15.9 & 15.5 & 12.5 & 12.9 & 14.0 & 13.4 & 15.0 & 21.7 & 29.3 \\
\hline F28 & 12-Methylhexadecanoic acid & 1981 & 1.4 & $?$ & $?$ & 0.7 & $\operatorname{tr}$ & $\operatorname{tr}$ & 1.1 & & & 1.3 \\
\hline F29 & 15-Methylhexadecanoic acid & 1994 & 1.2 & $\operatorname{tr}$ & $\operatorname{tr}$ & $\operatorname{tr}$ & $\operatorname{tr}$ & $\operatorname{tr}$ & $\operatorname{tr}$ & 0.8 & 0.6 & 1.6 \\
\hline F30 & 14-Methylhexadecanoic acid & 2002 & 25.4 & 25.8 & 19.6 & 19.9 & 22.5 & 20.2 & 20.4 & 28.6 & 27.1 & 56.9 \\
\hline F31 & 9-Heptadecenoic acid ${ }^{\mathrm{a}}$ & 2007 & 1.3 & $\operatorname{tr}$ & 2.7 & 1.1 & 2.4 & $\operatorname{tr}$ & 0.9 & & & 2.2 \\
\hline F32 & 11-Heptadecenoic acida & 2007 & & & & & & & & & & \\
\hline F33 & 2,10-Dimethylhexadecanoic acid & 2013 & 1.2 & $\operatorname{tr}$ & $\operatorname{tr}$ & 1.1 & 1.3 & 1.7 & 1.6 & 1.1 & 2.1 & 3.7 \\
\hline F34 & 4,10-Dimethylhexadecanoic acid & 2200 & 1.5 & $\operatorname{tr}$ & 1.6 & 1.6 & 1.5 & 2.5 & 2.1 & 1.4 & 1.9 & 5.0 \\
\hline F35 & 4,12-Dimethylhexadecanoic acid & 2029 & $\operatorname{tr}$ & 2.2 & $\operatorname{tr}$ & 1.8 & $\operatorname{tr}$ & 1.7 & 1.6 & & & \\
\hline F36 & Heptadecanoic acid & 2031 & 2.5 & $\operatorname{tr}$ & 1.7 & $\operatorname{tr}$ & 1.9 & $\operatorname{tr}$ & $\operatorname{tr}$ & 1.2 & 1.0 & 4.7 \\
\hline F37 & 2,14-Dimethylheptadecanoic acid & 2035 & 0.7 & $\operatorname{tr}$ & $\operatorname{tr}$ & 0.8 & 0.9 & 1.1 & 1.2 & 0.9 & 0.9 & 2.8 \\
\hline F38 & 10,14-Dimethylhexadecanoic acid & 3041 & 5.2 & 6.3 & 5.7 & 5.1 & 6.5 & 7.0 & 7.1 & 4.4 & 5.1 & 16.8 \\
\hline F39 & 4,14-Dimethylhexadecanoic acid & 2051 & 2.5 & 3.7 & 2.7 & 2.8 & 2.4 & 3.8 & 3.6 & 1.9 & 2.4 & 8.5 \\
\hline F40 & 4,8,12-Trimethylhexadecanoic acid & 2060 & 0.7 & $\operatorname{tr}$ & 0.7 & 0.6 & $\operatorname{tr}$ & 0.9 & 1.0 & 0.2 & 0.3 & 2.4 \\
\hline F41 & 2-Methylheptadecanoic acid & 2063 & 0.8 & $\operatorname{tr}$ & $\operatorname{tr}$ & $\operatorname{tr}$ & 1.1 & $\operatorname{tr}$ & 0.8 & 0.4 & 0.5 & 1.8 \\
\hline F42 & 10-Methylheptadecanoic acid & 2072 & 7.9 & 6.7 & 7.7 & 4.3 & 11.6 & 6.9 & 7.1 & 4.5 & 4.5 & 15.4 \\
\hline F43 & 12-Methylheptadecanoic acid & 2078 & 4.3 & $\operatorname{tr}$ & $\operatorname{tr}$ & $\operatorname{tr}$ & 4.9 & $\operatorname{tr}$ & $\operatorname{tr}$ & & & 10.6 \\
\hline F44 & 4-Methylheptadecanoic acid & 2079 & 4.4 & 9.4 & 9.2 & 7.0 & 5.6 & 9.8 & 9.7 & 7.4 & 9.4 & 10.8 \\
\hline F45 & 4,10,14-Trimethylhexadecanoic acid & 2085 & $?$ & 10.9 & 5.0 & 2.9 & 1.3 & 3.2 & $\operatorname{tr}$ & & & \\
\hline F47 & 14-Methylheptadecanoic acid & 2089 & 18.6 & 19.4 & 16.1 & 17.0 & 16.7 & 18.1 & 18.5 & 15.4 & 16.2 & 47.9 \\
\hline F48 & Octadeca-9,12-dienoic acid & 2099 & 1.9 & $\operatorname{tr}$ & 3.9 & & 2.8 & 1.3 & 1.3 & 1.0 & 0.8 & 2.3 \\
\hline F49 & 9-Octadecenoic acid & 2105 & 13.4 & 13.3 & 22.4 & 10.1 & 22.2 & 9.5 & 9.5 & 21.5 & 14.5 & 21.2 \\
\hline F50 & 11-Octadecenoic acid & 2110 & 5.9 & 7.7 & 6.9 & 4.1 & 7.5 & 4.7 & 4.6 & 9.5 & 7.2 & 10.4 \\
\hline F51 & 2,14-Dimethylheptadecanoic acid & 2119 & $\operatorname{tr}$ & & & & & & & & 1.4 & \\
\hline F52 & 4,10-Dimethylheptadecanoic acid & 2120 & $\operatorname{tr}$ & $\operatorname{tr}$ & $\operatorname{tr}$ & 1.2 & 1.3 & 1.5 & 1.6 & 2.4 & 2.9 & 4.2 \\
\hline F53 & 10,14-Dimethylheptadecanoic acid & 2124 & 3.0 & 3.4 & 3.5 & 3.3 & 4.0 & 4.8 & 4.9 & & & 11.1 \\
\hline F54 & Octadecanoic acid & 2131 & 2.7 & 2.3 & 1.6 & 2.1 & 1.8 & 1.2 & 1.3 & 2.8 & 1.1 & 4.1 \\
\hline F55 & 4,14-Dimethylheptadecanoic acid & 2137 & 1.8 & 2.1 & 2.0 & 2.2 & 2.2 & 3.1 & 3.2 & 2.4 & 2.2 & 7.2 \\
\hline
\end{tabular}


Table 1 (continued)

\begin{tabular}{|c|c|c|c|c|c|c|c|c|c|c|c|c|}
\hline & & RI & $\begin{array}{l}\text { M1 } \\
\delta^{\star}\end{array}$ & $\begin{array}{l}\text { M2 } \\
\sigma^{2}\end{array}$ & $\begin{array}{l}\text { M3 } \\
\delta\end{array}$ & $\begin{array}{l}\text { F1 } \\
\text { 우 }\end{array}$ & $\begin{array}{l}\text { F2 } \\
\text { 우 }\end{array}$ & $\begin{array}{l}\text { F3 } \\
\text { 우 }\end{array}$ & $\begin{array}{r}\text { F4 } \\
\text { 우 }\end{array}$ & $\begin{array}{r}\text { F5 } \\
\text { 우 }\end{array}$ & $\begin{array}{r}\mathrm{F} 6 \\
\text { 우 }\end{array}$ & $\begin{array}{l}\text { F7 } \\
\text { 우 }\end{array}$ \\
\hline F56 & Methyloctadecenoic acid & 2151 & 1.1 & $\operatorname{tr}$ & 1.0 & 0.9 & 1.6 & 1.5 & 0.8 & 0.3 & 0.3 & 1.7 \\
\hline F57 & Trimethylheptadecanoic acid & 2155 & & & & & & & & 0.6 & 0.4 & 1.9 \\
\hline F58 & 12-Methyloctadecanoic acid & 2175 & 3.5 & 3.1 & 3.2 & 2.4 & 4.9 & 3.1 & 3.4 & 5.2 & 2.4 & 6.5 \\
\hline F59 & 4-Methyloctadecanoic acid & 2179 & 0.6 & 0.5 & 0.4 & 0.3 & 0.5 & 1.3 & 0.9 & 1.2 & 0.8 & 1.1 \\
\hline F60 & 14-Methyloctadecanoic acid & 2183 & 20.9 & 22.2 & 18.4 & 19.6 & 21.6 & 22.1 & 21.9 & 29.5 & 23.3 & 46.0 \\
\hline F61 & 16-Methyloctadecanoic acid & 2203 & 2.9 & 7.0 & 3.1 & 2.3 & 5.1 & 2.1 & 2.6 & 8.9 & & 6.6 \\
\hline F62 & 9,10,11-Nonadecenoic acid & 2207 & 1.8 & 2.2 & 4.1 & 0.9 & 6.3 & 1.1 & 1.2 & 6.9 & 1.9 & 2.7 \\
\hline F63 & 10,14-Dimethyloctadecanoic acida & 2216 & 3.3 & 3.0 & 3.9 & 3.2 & 5.2 & 4.9 & 5.0 & 9.2 & 3.5 & 10.0 \\
\hline F64 & 8,14-Dimethyloctadecanoic acid & 2216 & & & & & & & & & & \\
\hline F65 & 6,14-Dimethyloctadecanoic acid & 2222 & & & & & & & & & & 1.9 \\
\hline F66 & 4,14-Dimethyloctadecanoic acid & 2230 & 3.0 & 3.5 & 2.8 & 2.8 & 4.8 & 3.9 & 3.8 & 7.2 & 2.5 & 8.1 \\
\hline F67 & Arachidonic acid & 2264 & 1.9 & 2.2 & 5.3 & 1.8 & 7.6 & 3.5 & 4.4 & 3.4 & 1.5 & 6.0 \\
\hline F68 & 10-Methylnonadecanoic acid & 2269 & 1.1 & $\operatorname{tr}$ & 1.5 & $\operatorname{tr}$ & 2.8 & $\operatorname{tr}$ & 1.3 & 4.1 & 1.4 & 3.9 \\
\hline F69 & 14-Methylnonadecanoic acid & 2278 & 2.2 & 3.2 & 2.5 & 2.0 & 4.4 & 3.2 & 3.2 & 6.0 & 1.7 & 6.8 \\
\hline F70 & 16-Methylnonadecanoic acid & 2290 & 1.6 & $\operatorname{tr}$ & 1.0 & 0.9 & 1.9 & 1.0 & 1.2 & 5.8 & 1.1 & 5.2 \\
\hline F71 & 11-Icosenoic acid & 2306 & 5.3 & 4.0 & 7.2 & 1.6 & 10.9 & 2.8 & 2.8 & 11.9 & & 7.0 \\
\hline F72 & 12-Icosenoic acid & 2309 & 4.3 & 4.7 & 4.5 & 3.2 & 5.3 & 3.9 & 3.7 & 5.7 & 7.4 & 5.9 \\
\hline F73 & 13-Icosenoic acid ${ }^{a}$ & 2313 & 6.0 & 5.9 & 6.2 & 2.9 & 9.9 & 5.3 & 4.8 & 12.7 & 6.1 & 7.2 \\
\hline F74 & 4,X-Dimethylicosanoic acid & 2313 & & & & & & & & & & \\
\hline F75 & 8,14-Dimethylnonadecanoic acid & 2321 & $\operatorname{tr}$ & & & & & & & & & 1.3 \\
\hline F76 & Icosanoic acid & 2331 & 10.7 & 6.5 & 5.2 & 6.0 & 7.8 & 5.5 & 5.7 & 11.1 & 2.5 & 10.5 \\
\hline F77 & 8-Methylicosanoic acid & 2367 & 4.5 & 5.1 & 4.0 & 2.9 & 8.1 & 5.1 & 4.6 & 7.6 & 1.7 & 7.3 \\
\hline F78 & 14-Methylicosanoic acid & 2373 & 1.9 & 7.5 & 2.7 & 2.4 & 3.5 & 3.9 & 2.6 & 4.2 & 1.2 & 5.7 \\
\hline F79 & 16-Methylicosanoic acid & 2381 & 7.5 & 8.2 & 6.4 & 7.4 & 8.5 & 7.0 & 7.2 & 7.9 & 3.2 & 14.0 \\
\hline F80 & 13-Henicosenoic acid & 2408 & 18.3 & 23.2 & 25.0 & 13.7 & 29.0 & 18.3 & 18.7 & 21.0 & 15.9 & 29.2 \\
\hline F81 & 12-Henicosenoic acida & 2408 & & & & & & & & & & \\
\hline F82 & Henicosanoic acid & 2431 & 6.1 & 3.6 & 3.2 & 4.1 & 4.7 & 3.2 & 3.6 & 4.7 & 1.0 & 4.9 \\
\hline F83 & 8-Methylhenicosanoic acid & 2468 & 4.4 & 4.2 & 3.9 & 3.5 & 6.4 & 4.0 & 4.2 & 7.1 & 1.2 & 6.5 \\
\hline F84 & 14-Methylhenicosanoic acid & 2472 & & & & & & & & & 1.9 & 3.9 \\
\hline F85 & 16-Methylhenicosanoic acid & 2475 & $\operatorname{tr}$ & ? & $\operatorname{tr}$ & $\operatorname{tr}$ & $\operatorname{tr}$ & 1.3 & 1.0 & 10.3 & 0.6 & 8.6 \\
\hline F86 & 12,16-Docosadienoic acid & 2499 & 5.2 & 4.1 & 3.5 & 3.2 & 3.4 & 5.7 & 4.8 & 7.8 & 2.3 & 5.2 \\
\hline F87 & 13-Docosenoic acid & 2516 & 100 & 100 & 100 & 100 & 100 & 100 & 100 & 100 & 100 & 100 \\
\hline F88 & 15-Docosenoic acid & 2524 & 18.0 & 21.1 & 18.5 & 19.7 & 19.1 & 19.0 & 18.5 & 19.6 & 22.8 & 22.0 \\
\hline F89 & 14-Docosenoic acida & 2524 & & & & & & & & & & \\
\hline F90 & Docosanoic acid & 2532 & 23.5 & 10.7 & 8.9 & 17.0 & 10.0 & 12.7 & 13.1 & 6.8 & 3.7 & 9.0 \\
\hline F91 & 8-Methyl-7-docosenoic acid & 2546 & 1.8 & 4.9 & 1.8 & 1.9 & 2.4 & 2.3 & 2.5 & 1.7 & & 2.1 \\
\hline F92 & 8-Methyl-13-docosenoic acid & 2554 & 1.5 & & 1.5 & 1.9 & 1.8 & 1.7 & 1.7 & & & \\
\hline F93 & $X$-Methyldocosanoic acid & 2573 & & & & & & & & 1.6 & & 1.1 \\
\hline F94 & 8-Methyldocosanoic acid & 2584 & 3.2 & 7.9 & 3.5 & 3.7 & 3.8 & 3.0 & 3.1 & & & 1.8 \\
\hline F95 & 13-Tricosenoic acida & 2610 & $\operatorname{tr}$ & $?$ & 5.3 & $\operatorname{tr}$ & 5.5 & $\operatorname{tr}$ & 5.1 & & & 4.9 \\
\hline F96 & 14-Tricosenoic acid ${ }^{a}$ & 2610 & & & & & & & & & & \\
\hline F97 & 15-Tricosenoic acid & 2612 & 23.0 & 23.7 & 18.3 & 25.0 & 18.0 & 21.5 & 16.9 & 10.7 & 6.1 & 18.0 \\
\hline F98 & Tricosanoic acid & 2631 & 1.5 & 2.3 & & 1.1 & 1.1 & 1.5 & 1.5 & & & \\
\hline F99 & Tetracosa-7,15-dienoic acid & 2667 & 3.8 & $?$ & 2.6 & 2.8 & $?$ & $?$ & $\operatorname{tr}$ & 4.4 & 0.9 & 1.7 \\
\hline F100 & Tetracosadienoic acid ${ }^{\mathrm{b}}$ & 2669 & 4.1 & $?$ & $\operatorname{tr}$ & 4.8 & 3.0 & 3.7 & 2.6 & & 1.7 & 1.9 \\
\hline F101 & Tetracosa-13,17-dienoic acid & 2674 & 6.2 & 47.1 & 23.1 & 5.6 & $\operatorname{tr}$ & $?$ & 3.7 & 0.7 & & 1.1 \\
\hline F104 & Tetracosadienoic acid & 2681 & 5.0 & 3.3 & 3.1 & 4.1 & 2.8 & 3.4 & 2.4 & 1.6 & 0.9 & 2.5 \\
\hline F105 & 15-Tetracosenoic acid & 2694 & 83.7 & 68.3 & 72.6 & 99.0 & 69.9 & 74.5 & 74.8 & 43.1 & 33.0 & 62.1 \\
\hline F106 & Tetracosanoic acid & 2732 & 2.6 & $\operatorname{tr}$ & $\operatorname{tr}$ & 1.9 & 1.2 & 1.5 & 0.9 & 0.8 & 0.9 & 0.8 \\
\hline
\end{tabular}

RI, retention index of the respective methyl ester; Tr, trace component. Absence of entries denotes not detectable above detection limit $(0.1 \%)$. ${ }^{a}$ Compounds elute together with previous entry in the table. The percentages given represent the amount for both compounds. ${ }^{b}$ A group of at least four, probably more acids. The overlapping peaks of the different derivatives did not allow full structural assignment. Two major components are 7,17-tetracosadienoic acid, eluting earlier, and 13,17-tetracosadienoic acid, eluting later. 
<smiles>CCCCCCCCCCCCC(C)CCC(=O)O</smiles>

F27<smiles>CCCCCCC(C)CCCCCCCCC(=O)O</smiles>

F26<smiles>CCC(C)CCCCCCCCCCCCC(=O)O</smiles>

F30<smiles>CCC(C)CCCC(C)CCCCCCCCC(=O)O</smiles>

F38<smiles>CCCCC(C)CCCCCCCCCC(C)CCC(=O)O</smiles>

F66<smiles>CCCCC/C=C/C/C=C/C/C=C/C/C=C/CCCC(=O)O</smiles>

F67<smiles>CCCCCCCC/C=C/CCCCCCCCCC(=O)O</smiles>

F71<smiles>CCCCCCCC/C=C/CCCCC(C)CCCCCCC(=O)O</smiles>

F92<smiles>CCCCCCCC/C=C/CCCCCCCCCCCCCC(=O)O</smiles><smiles>CCCCCCCC/C=C/CCCCCC/C=C/CCCCCC(=O)O</smiles>

Figure 5: Representative structures for various types of acids found in Loxocemus bicolor. The Z-configuration of the double bonds is tentative.

\section{Discussion}

Carboxylic acids evidently are ubiquitous scent gland products of snakes, having been documented in colubrids [12], boids [6, 8, 12], pythonids [12], elapids [7], viperids [9, 10], and leptotyphlopids [5]. These compounds typically possess 2-26 carbon atoms and chiefly feature saturated or monounsaturated straight chains. Lower molecular weight acids $\left(\mathrm{C}_{4}\right.$ and $\left.\mathrm{C}_{5}\right)$ with a single methyl branch [8, 12], hydroxypropanoic acid [6, 8], methylbenzoic acid [9], and phenylacetic as well as phenylpropanoic acids [7-9] also have been documented in some species. The fatty acids of Loxocemus bicolor include some of the straightchain compounds reported in other snakes, but distinctly contain more than 65 mono-, di-, and trimethyl-branched compounds, and many compounds with one, two, or three double bonds. The significance of this structural diversity is open to speculation. The Texas blindsnake (Leptotyphlops dulcis), another fossorial snake, discharges repellent cloacal fluids, including scent gland secretions, when attacked by ants [33]. Blum et al. [5] suggested that fatty acids in the secretions of L. dulcis, which they identified as 13 straight-chain $\mathrm{C}_{12}$ to $\mathrm{C}_{20}$ compounds, act against ants as insecticides or by exploiting out-of-context semiochemical responses. Fatty acids of $L$. bicolor should be tested for similar allomonal properties against ants and other offending leaf-litter arthropods.

Glyceryl monoethers possessing $n$-alkyl residues ranging in carbon-chain length from 12 to 20, chiefly $\mathrm{C}_{14}, \mathrm{C}_{16}$, and $\mathrm{C}_{18}$ chains, were reported in the scent gland secretions of male and female western diamondback rattlesnakes (Crotalus atrox) [11]. Young et al. [34]., however, failed to observe bands corresponding to this compound class in thin-layer chromatograms of the secretions of two pitvipers (Crotalinae), the eastern diamondback rattlesnake (C. adamanteus) and the Florida cottonmouth (Agkistrodon piscivorus conanti). Our analysis of L. bicolor affirms 43 glyeryl alkyl monoethers as scent gland products, revealing $\mathrm{C}_{13}$ to $\mathrm{C}_{24}$ straight-chain or methyl-branched alkyl chains. Glyceryl ethers, mostly showing saturated $\mathrm{C}_{15}$ to $\mathrm{C}_{22} n$-alkyl chains, have also been observed in the femoral gland secretions of a lacertid lizard (Acanthodactylus boskianus) [32]. These compounds may occur widely among squamate reptiles.

We observed straight chain alcohols in the scent gland secretions of L. bicolor ranging in carbon-chain length from 13 to 24 . This compound class has not previously been described from snake scent glands. However, 
<smiles>CCOSC(=O)CC</smiles>

11<smiles>CCOC(=O)C(C)C(=O)O[Na]</smiles>

14<smiles>CCCCCCCC(C)C</smiles><smiles>O=C(CC(=O)SCCCCCCCO)O[Na]</smiles>

15<smiles>CCCCCCCCCCCCCCCCCC(=O)O</smiles>

13

$\Delta 11$-Desaturase

11-Desaturase<smiles>CCCCCCCC/C=C\CCCCCCCC(=O)O</smiles>

16<smiles>O=C(O)CCC(=O)O[Na]</smiles>

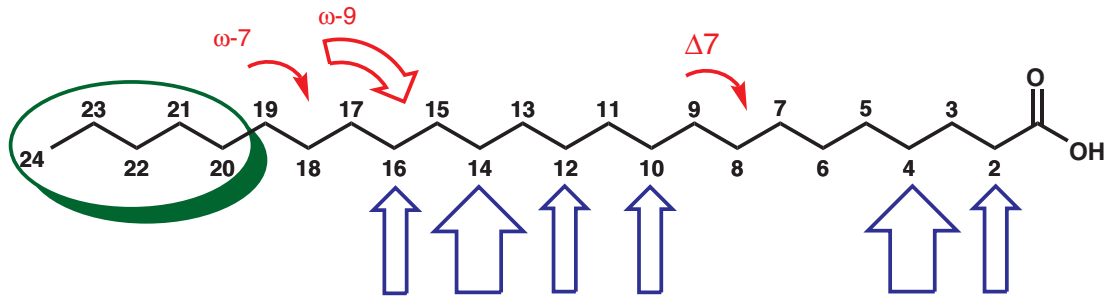

17

Figure 6: Biosynthetic features leading to a diversity of fatty acids. Black: standard biosynthetic pathway to saturated and unsaturated fatty acids. Blue: the incorporation of methylmalonate (14) leads to methyl groups at certain positions of the chain. Red: additional oxidation leads to double bonds at preferentially at $\omega-9$ and $\omega$-7. Dienoic acids are formed by an additional double-bond introduced, e.g., at $\Delta 7$. Green: The chain length can vary because of additional chain elongation with malonate (15). Odd numbered acids can be formed from a propionate starter (11) or $\alpha$-oxidation of an acid (not shown). Thickness of arrows in $\mathbf{1 7}$ indicated relative importance of the modification at a certain position.

alcohols possessing chains of more than 30 carbons have been observed in extracts of the shed or intact epidermis of colubrid snakes [35, 36]. Such long-chain compounds are among the nonpolar lipids that may contribute to the transepidermal water barrier of the epidermis. The significance of alcohols in the scent gland secretions is unclear.

\section{Experimental part}

\subsection{Methods}

Scent gland secretions were collected from three male and seven female snakes (total lengths $=61-127 \mathrm{~cm}$ ) maintained on rodents at the Memphis Zoo (TN, USA) and Vida Preciosa International, Inc. (Boerne, TX, USA). Most specimens were captured in Central America, possibly Honduras; two females were reared in captivity from these wild-caught individuals. Snakes were restrained while manual pressure was applied to the base of the tail. The emerging stream of scent gland fluids was directed into glass vials to which several milliliters of dichloromethane was added. Samples were kept frozen until analysis.

\subsection{GC/MS analyses}

GC/MS analyses were performed on an HP7890A GC connected to an HP5975C mass selective detector fitted with an HP-5ms fused silica capillary column (30 m, $0.22 \mathrm{~mm}$ i.d., $0.25 \mu \mathrm{m}$ film, Agilent Technologies, USA). Helium served as carrier gas. Conditions were as follows: injection volume $1 \mu \mathrm{L}$, transfer line $300{ }^{\circ} \mathrm{C}$, injector $250^{\circ} \mathrm{C}$, electron energy $70 \mathrm{eV}$. Linear retention indices were determined from a homologous series of $n$-alkanes $\left(\mathrm{C}_{8}-\mathrm{C}_{32}\right)$.

\subsection{Derivatizations}

\subsubsection{Silylation}

$N$-methyl- $N$-(trimethylsilyl)trifluoroacetamide

(MSTFA) $(50 \mu \mathrm{L})$ was added to $100 \mu \mathrm{L}$ of an extract, followed by 

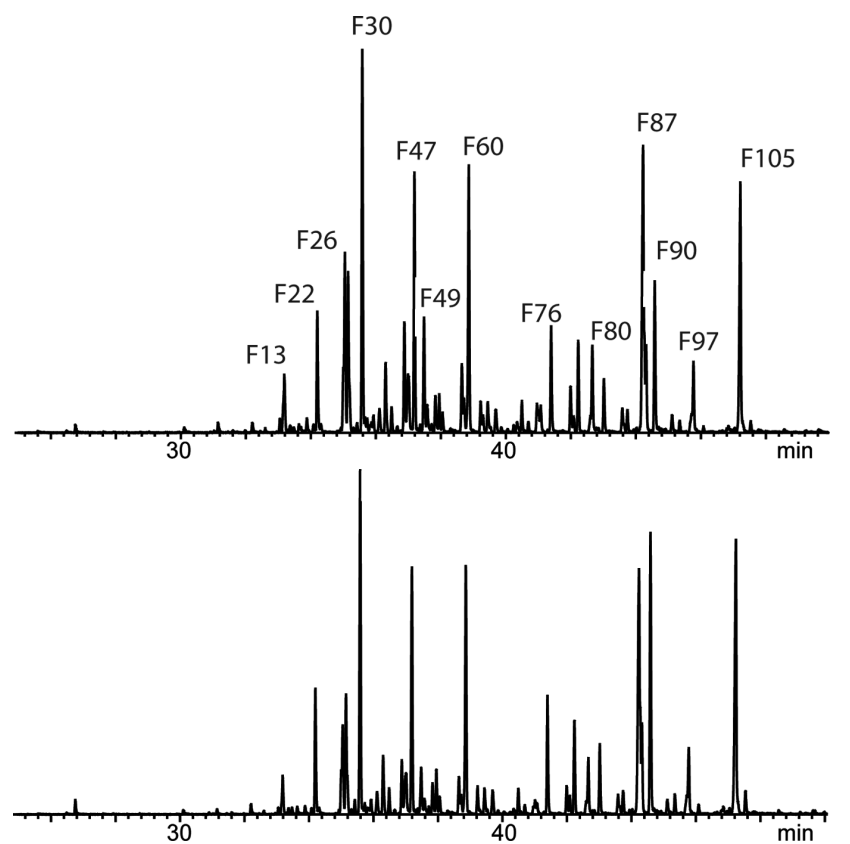

Figure 7: Fatty acids detected in scent gland secretions of male $M 3$ (upper trace) and female F1 (lower trace) Loxocemus bicolor. The extract was methylated to convert acids into methyl esters. The ion trace $m / z 74$, characteristic for methyl esters of fatty acids, is shown to exclude other compounds.

heating for $1 \mathrm{~h}$ at $60{ }^{\circ} \mathrm{C}$. Excess MSTFA and solvent were removed under a gentle stream of nitrogen to a volume of $5 \mu \mathrm{L}$. Finally, dichloromethane was added $(100 \mu \mathrm{L})$ and the derivatized extract was analysed by GC/MS.

\subsubsection{Methylation}

$N$-methyl- $N$-nitroso- $p$-toluenesulfonamide $(0.41 \mathrm{M}$ in 1:1 diethyl ether/diethylethylenglykol monoethyl ether, 1 eq.) was added to a 5-mL vial equipped with a Teflon-lined septum cap carrying a small teflon tube as gas outlet. The tubing was connected to a second vial serving as gas washer. This vial was empty and carried a second Teflon tube as gas outlet. This tube was connected to a third vial, carrying diethyl ether. The latter vial was cooled with ice. $\mathrm{KOH}$ (1.1 eq, $0.37 \mathrm{M}$ solution in 1:1 methanol/water) was added to the first tube by a syringe. The produced yellow was trapped in the diethyl ether vial. Subsequently, the yellow solution was added to $100 \mu \mathrm{L}$ of an extract until the gas formation ceased. The derivatized extract was analyzed by GC/MS. Caution: Pure diazomethane is explosive.

\subsubsection{3-Methylpyridyl esters}

About $100 \mu \mathrm{L}$ dichloromethane extract was treated with $20 \mu \mathrm{L}$ oxalyl chloride for 1 day in a 2-mL vial. Excess oxalyl chloride was removed by evaporation with a stream of nitrogen. One drop 3-pyridinemethanol and $100 \mu \mathrm{L}$ dichloromethane were added. The mixture was heated for $1 \mathrm{~h}$ in a heating block at $60^{\circ} \mathrm{C}$, followed by GC/MS analysis.

\subsubsection{Pyrrolidides}

About $100 \mu \mathrm{L}$ dichloromethane extract was treated with $50 \mu \mathrm{L}$ of a 9:1 mixture of pyrrolidine and pyridine in a 2 -mL vial. After $1 \mathrm{~h}$ at room temperature, the $100 \mu \mathrm{L}$ saturated $\mathrm{NaHCO}_{3}$ solution was added. The organic phase was removed, dried with molecular sieve, and analyzed by GC/MS.

\subsubsection{Dimethyl disulfide derivatives}

Dimethyl disulfide (DMDS) adducts were obtained by stirring equal amounts of freshly distilled DMDS and
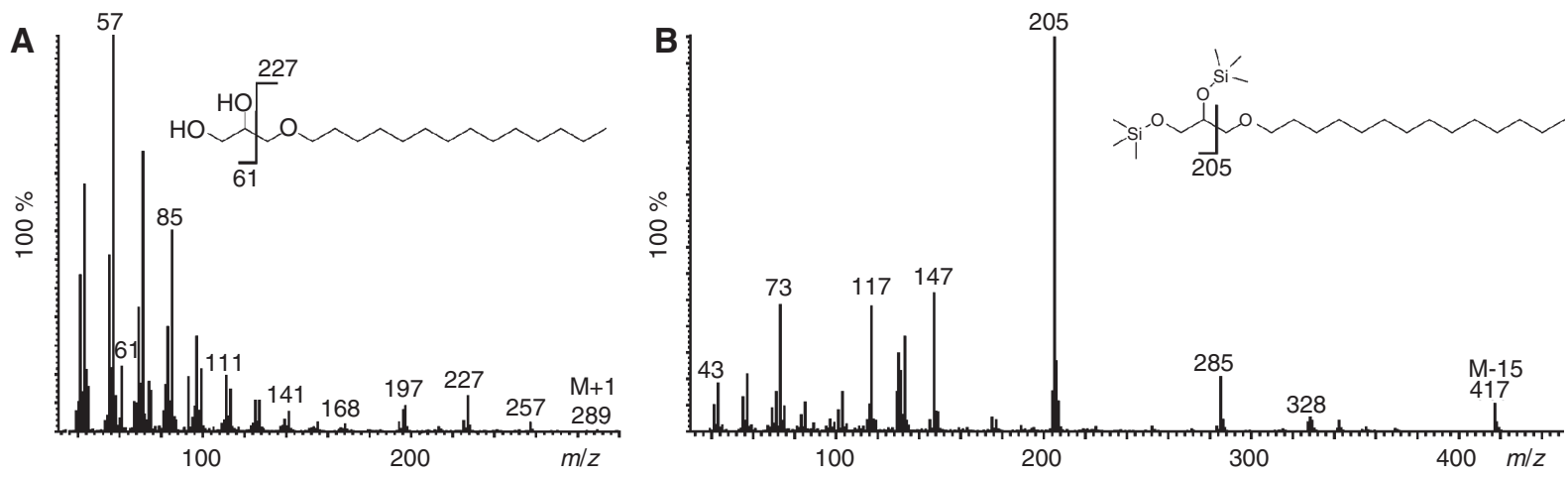

Figure 8: Mass spectra of 1-0-Tetradecylglyceride (A) and its bistrimethylsilyl derivative (B). Characteristic mass spectrometric cleavages are indicated. 
Table 2: 1-O-Alkylglycerols in the scent gland secretions of Loxocemus bicolor.

\begin{tabular}{|c|c|c|c|}
\hline Number & Alkyl residue & $I$ & Conc. \\
\hline G1 & Tridecyl & 2212 & $x x x$ \\
\hline $\mathrm{G} 2$ & 12-Methyltridecyl & 2280 & $x$ \\
\hline G3 & Tetradecyl & 2312 & $x x x$ \\
\hline G4 & $X$-Methyltetradecyl & 2338 & $x$ \\
\hline G5 & 13-Methyltetradecyl & 2365 & $x$ \\
\hline G6 & 12-Methyltetradecyl & 2375 & $x$ \\
\hline G7 & Pentadecyl & 2403 & $x x x$ \\
\hline G8 & X-Methylpentadecyl & 2432 & $x$ \\
\hline G9 & $X$-Methylpentadecyl & 2435 & $x$ \\
\hline G10 & 14-Methylpentadecyl & 2460 & $x$ \\
\hline G11 & 13-Methylpentadecyl & 2471 & $x$ \\
\hline G12 & Hexadecyl & 2499 & $x x x$ \\
\hline G13 & 14-Methylhexadecyl & 2568 & $x x x$ \\
\hline G14 & Heptadecyl & 2594 & $x$ \\
\hline G15 & X-Methylheptadecyl & 2618 & $x$ \\
\hline G16 & $X$-Methylheptadecyl & 2637 & $x$ \\
\hline $\mathrm{G} 17$ & $X$-Methylheptadecyl & 2642 & $x$ \\
\hline G18 & 16-Methylheptadecyl & 2648 & $x$ \\
\hline G19 & 15-Methylheptadecyl & 2664 & $x$ \\
\hline $\mathrm{G} 20$ & Octadecenyl & 2667 & $x$ \\
\hline $\mathrm{G} 21$ & Octadecyl & 2689 & $x x$ \\
\hline $\mathrm{G} 22$ & X-Methyloctadecyl & 2717 & $x$ \\
\hline G23 & $X$-Methyloctadecyl & 2727 & $x$ \\
\hline $\mathrm{G} 24$ & X-Methyloctadecyl & 2727 & $x$ \\
\hline $\mathrm{G} 25$ & X-Methyloctadecyl & 2737 & $x$ \\
\hline $\mathrm{G} 26$ & 16-Methyloctadecyl & 2759 & $x$ \\
\hline $\mathrm{G} 27$ & Nonadecyl & 2785 & $x x$ \\
\hline $\mathrm{G} 28$ & X-Methylnonadecyl & 2799 & $x$ \\
\hline G29 & 18-Methylnonadecyl & 2827 & $x$ \\
\hline G30 & Icosenyl & 2858 & $x$ \\
\hline G31 & Icosenyl & 2862 & $x$ \\
\hline G32 & Icosenyl & 2866 & $x$ \\
\hline G33 & Icosyl & 2883 & $x x$ \\
\hline G34 & $X$-Methylicosyl & 2933 & $x$ \\
\hline G35 & Henicosyl & 2977 & $x$ \\
\hline G36 & Docosenyl & 3052 & $x$ \\
\hline G37 & Docosenyl & 3055 & $x$ \\
\hline G38 & Docosenyl & 3058 & $x$ \\
\hline G39 & Docosenyl & 3061 & $x$ \\
\hline G40 & Docosyl & 3075 & $x$ \\
\hline G41 & Tricosenyl & 3150 & $x$ \\
\hline G42 & Tetracosadienyl & 3233 & $x$ \\
\hline G43 & Tetracosenyl & 3247 & $x x$ \\
\hline
\end{tabular}

Alkyl residue: Aliphatic part of the ether; I, gas chromatographic retention index; conc., relative concentration: $\mathrm{xxx}$, major component; $x x$, minor component; and $x$, trace component.

$100 \mu \mathrm{L}$ of a natural extract with $5 \mu \mathrm{L}$ of a $5 \% \mathrm{I}_{2}$-solution in diethyl ether at $60{ }^{\circ} \mathrm{C}$ overnight. Then excess $\mathrm{I}_{2}$ was removed with saturated aqueous $\mathrm{Na}_{2} \mathrm{~S}_{2} \mathrm{O}_{3}$. The organic phase was separated and the aqueous phase was extracted twice with $100 \mu \mathrm{L}$ pentane. The combined organic phases were dried with $\mathrm{NaCl}$ and reduced to a

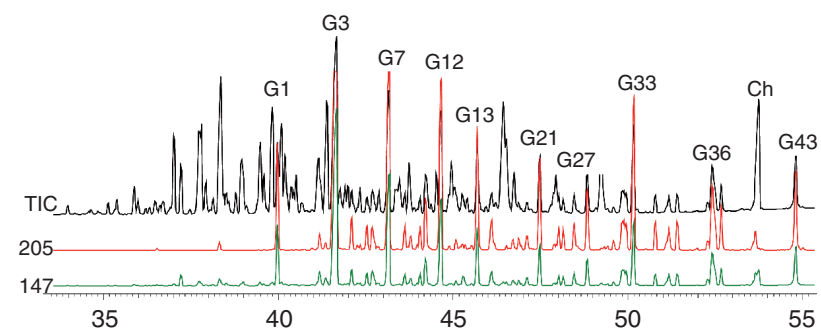

Figure 9: TIC of a scent gland secretion extract of Loxocemus bicolor silylated with MSTFA. Ions traces characteristic of 1-O-alkylglycerols, $m / z 147$ and $m / z$ 205, are shown. Important peaks are annotated according toTable 2 . Ch, cholesterol.

volume of $50 \mu \mathrm{L}$. The derivatized extract was then analyzed by GC/MS.

Acknowledgment: S. Reichling (Memphis Zoo, TN, USA) and D. and T. Barker (Vida Preciosa International, Inc., Boerne, TX, USA) made specimens available for our study.

\section{References}

1. Weldon PJ, Flachsbarth B, Schulz S. Natural products from the integument of nonavian reptiles. Nat Prod Rep 2008;25:738-56.

2. Greene MJ, Mason RT. The effects of cloacal secretions on brown tree snake behavior. In: Mason RT, LeMaster MP, MüllerSchwarze D, editors. Chemical signals in vertebrates 10 . New York: Springer, 2005:49-55.

3. Greene MJ, Mason RT. Pheromonal inhibition of male courtship behaviour in the brown tree snake, Boiga irregularis: a mechanism for the rejection of potential mates. Anim Behav 2003;65:905-10.

4. Weldon PJ, Leto TL. A comparative analysis of proteins in the scent gland secretions of snakes. J Herpetol 1995;29:474-6.

5. Blum MS, Byrd JB, Travis JR, Watkins II JF, Gehlbach FR. Chemistry of the cloacal sac secretion of the blind snake Leptotyphlops dulcis. Comp Biochem Physiol B 1971;38:103-7.

6. Simpson JT, Weldon PJ, Sharp TR. Identification of major lipids from the scent gland secretions of Dumeril's ground boa (Acrantophis dumerili Jan) by gas chromatography-mass spectrometry. Z Naturforsch Sect C J Biosci 1988;43:914-7.

7. Weldon PJ, Sampson HW, Wong L, Lloyd HA. Histology and biochemistry of the scent glands of the yellow-bellied sea snake (Pelamis platurus: Hydrophiidae). J Herpetol 1991;25:367-70.

8. Simpson JT, Sharp TR, Wood WF, Weldon PJ. Further analysis of lipids from the scent gland secretions of Dumeril's ground boa (Acrantophis dumerili Jan). Z Naturforsch Sect C J Biosci 1993;48:953-5.

9. Weldon PJ, Ortiz R, Sharp TR. The chemical ecology of crotaline snakes. In: Campbell JA, Brodie Jr ED, editors. Biology of the pitvipers. Tyler, Tex.: Selva, 1992: 309-19.

10. Razakov RR, Sadykov AS. Study of complex-mixtures of natural substances by defocusing and DADI methods. VI. components of pre-anal gland secretion in some poisonous snakes. Khim Prir Soedin 1986;4:421-3. 
11. Weldon PJ, Lloyd HA, Blum MS. Glycerol monoethers in the scent gland secretions of the Western diamondback rattlesnake (Crotalus atrox; Serpentes, Crotalinae). Experientia 1990;46:774-5.

12. Wood WF, Parker JM, Weldon PJ. Volatile components in scent gland secretions of garter snakes (Thamnophis spp.). J Chem Ecol 1995;21:213-9.

13. Greene HW. Dietary correlates of the origin and radiation of snakes. Am Zoologist 1983;23:431-41.

14. Mora-Benavides JM. Predation by Loxocemus bicolor on the eggs of Ctenosaura similis and Iguana iguana. J Herpetol 1987;21:334-5.

15. Wiens JJ, Kuczynski CA, Smith SA, Mulcahy DG, Sites JW, Townsend TM, et al. Branch lengths, support, and congruence: testing the phylogenomic approach with 20 nuclear loci in snakes. Syst Biol 2008;57:420-31.

16. Pyron RA, Burbrink FT, Wiens JJ. A phylogeny and revised classification of Squamata, including 4161 species of lizards and snakes. BMC Evol Biol 2013;13:93.

17. Ryhage R, Stenhagen E. Mass spectrometric studies: IV. esters of monomethyl-substituted long chain carboxylic acids. Arkiv Kemi 1961;15:291-304.

18. Schulz S. Composition of the silk lipids of the spider Nephila clavipes. Lipids 2001;36:637-47.

19. Harvey DJ. Picolinyl esters as derivatives for the structural determination of long chain branched and unsaturated fatty acids. Biomed Mass Spectrom 1982;9:33-8.

20. Fay L, Richli U. Location of double bonds in polyunsaturated fatty acids by gas chromatography-mass spectrometry after 4,4-dimethyloxazoline derivatization. J Chromatogr A 1991;541:89-98.

21. Andersson BA, Holman RT. Pyrrolidides for mass spectrometric determination of the position of the double bond in monounsaturated fatty acids. Lipids 1974;9:185-90.

22. Scribe P, Guezennec J, Dagaut J, Pepe C, Saliot A. Identification of the position and the stereochemistry of the double bond in monounsaturated fatty acid methyl esters by gas chromatography/mass spectrometry of dimethyl disulfide derivatives. Anal Chem 1988;60:928-31.

23. Buser HR, Arn H, Guerin P, Rauscher S. Determination of double bond position in mono-unsaturated acetates by mass spectrometry of dimethyl disulfide adducts. Anal Chem 1983;55: 818-22.
24. Harvey DJ. Lipids from the guinea pig Harderian gland: use of picolinyl and other pyridine-containing derivatives to investigate the structures of novel branched-chain fatty acids and glycerol ethers. Biol Mass Spectrom 1991;20:61-9.

25. Christie WW, Brechany EY, Holman RT. Mass spectra of the picolinyl esters of isomeric mono- and dienoic fatty acids. Lipids 1987;22:224-8.

26. Yu QT, Liu BN, Zhang JY, Huang ZH. Location of methyl branchings in fatty acids: fatty acids in uropygial secretion of Shanghai duck by GC-MS of 4,4-dimethyloxazoline derivatives. Lipids 1988;23:804-10.

27. Zhang JY, Yu QT, Liu BN, Huang ZH. Chemical modification in mass spectrometry IV-2-alkenyl-4,4-dimethyloxazolines as derivatives for the double bond location of long-chain olefinic acids. Biol Mass Spectrom 1988;15:33-44.

28. Vetter W, Walther W, Vecchi M. Pyrrolidide als Derivate für die Strukturaufklärung aliphatischer und alicyclischer Carbonsäuren mittels Massenspektrometrie. Helv Chim Acta 1971;54: 1599-605.

29. Andersson BÅ. Mass spectrometry of fatty acid pyrrolidides. Prog Chem Fats Lipids 1978;16:279-308.

30. Nawrath T, Gerth K, Müller R, Schulz S. Volatile methyl esters of medium chain length from the bacterium Chitinophaga Fx7914. Chem Biodiversity 2010;7:2228-53.

31. Draffan GH, Stillwell RN, McCloskey JA. Electron-impact-induced rearrangement of trimethylsilyl groups in long chain compounds. Org Mass Spectrom 1968;1:669-85.

32. Khannoon ER, Flachsbarth B, El-Gendy A, Mazik K, Hardege JD, Schulz S. New compounds, sexual differences, and agerelated variations in the femoral gland secretions of the lacertid lizard Acanthodactylus boskianus. Biochem Syst Ecol 2011;39:95-101.

33. Gehlbach FR, Watkins JF, Reno HW. Blind snake defensive behavior elicited by ant attacks. BioScience 1968;18:784-5.

34. Young BA, Frazer BA, Fried B, Lee M, Lalor J, Sherma J. Determination of cloacal scent-gland lipids from two sympatric snakes, the eastern diamondback rattlesnake (Crotalus adamanteus) and the Florida cottonmouth (Agkistrodon piscivorus conanti). J Planar Chromatogr Mod TLC 1999;12:196-201.

35. Ahern DG, Downing DT. Skin lipids of the Florida indigo snake. Lipids 1974;9:8-14.

36. Mason RT. Chemical ecology of the red-sided garter snake, Thamnophis sirtalis parietalis. Brain Behav Evol 1993;41:261-8. 